\title{
Compositional and Material Properties of Rat Bone after Bisphosphonate and/or Strontium Ranelate Drug Treatment
}

\author{
Yuchin $\mathrm{Wu}^{\mathbf{a}}$, Samer M. Adeeb ${ }^{\mathbf{a}, \mathbf{b}}$, M. John Duke ${ }^{\mathbf{c}}$, David Munoz-Paniagua ${ }^{\mathrm{d}}$, Michael R. Doschak ${ }^{\mathbf{a}, \mathbf{e}}$ \\ ${ }^{a}$ Department of Biomedical Engineering, University of Alberta, Edmonton, Alberta, Canada \\ ${ }^{\mathbf{b}}$ Civil \& Environmental Engineering, University of Alberta, Edmonton, Alberta, Canada \\ ${ }^{\mathbf{c}}$ SLOWPOKE Reactor Facility, University of Alberta, Edmonton, Alberta, Canada \\ ${ }^{\mathbf{d}}$ National Research Council - National Institute for Nanotechnology, Edmonton, Alberta, Canada \\ ${ }^{\mathbf{e}}$ Faculty of Pharmacy \& Pharmaceutical Sciences, University of Alberta, Edmonton, Alberta, Canada
}

Received, December 7, 2012; Revised, January 15, 2013; Accepted, January 16, 2013; Published, January 16, 2013.

\begin{abstract}
Purpose. We investigated elemental strontium and/or bisphosphonate drug incorporation upon the compositional and biomechanical properties of vertebral bone, in a rat model of Osteoporosis secondary to ovariectomy. Methods. Six month old female rats were ovariectomized (OVX) and divided into untreated OVXVehicle, OVX-RIS (Risedronate bisphosphonate [BP] treated), OVX-SrR (Strontium Ranelate [Protos ${ }^{\circledR}$ ] treated), combination OVX-RIS+SrR, and sham-operated controls. After 16 weeks of treatment, rats were euthanized and lumbar vertebra were dissected. Micro-Computed Tomography (micro-CT), Electron Probe Micro-Analysis (EPMA), mechanical testing in compression and nano-indentation testing were then undertaken to evaluate bone morphometry, elemental composition, material properties and strength. Results. Bone Volume was significantly reduced in the OVX-Vehicle $\left(133 \pm 10 \mathrm{~mm}^{3}\right)$ compared with OVX-RIS $\left(169 \pm 22 \mathrm{~mm}^{3}\right)$, OVXSrR $\left(145 \pm 2 \mathrm{~mm}^{3}\right)$, and OVX-RIS+SrR $\left(172 \pm 8 \mathrm{~mm}^{3}\right)$. EPMA mapped elemental Sr deposition to the periosteal surface of cortical bone (50-100 $\mu \mathrm{m}$ thick), endosteal trabecular surfaces (20 $\mu \mathrm{m}$ thick), as well as to both vertebral growth plates. The atomic ratios of $(\mathrm{Ca}+\mathrm{Sr}) / \mathrm{P}$ were significantly reduced with $\mathrm{SrR}$ treatment $(2.4 \%$ $6.6 \%$ ), indicating $\mathrm{Sr}$ incorporation into bone mineral. No significant differences were measured in vertebral bone reduced modulus by nano-indentation. Conversely, all BP-dosed groups had significantly increased structural bone strength. Conclusions. Thus, we conclude that BP drugs dominate the conservation of trabecular geometry and structural strength in OP rats, whereas $\mathrm{Sr}$ drugs likely influence bone volume and material composition locally.
\end{abstract}

This article is open to POST-PUBLICATION REVIEW. Registered readers (see "For Readers") may comment by clicking on ABSTRACT on the issue's contents page.

\section{INTRODUCTION}

Osteoporosis (OP) related fractures affect one third of postmenopausal women worldwide, with an estimated 2 million OP related fractures occurring annually in the United States alone (1). Fracture occurs when bone encounters load of sufficient magnitude (and rate of loading) to exceed its mechanical strength. Currently, Bone Mineral Density (BMD) measurements derived from DualEnergy X-ray Analysis (DXA) are heavily relied upon in order to estimate bone strength for $\mathrm{OP}$ patients in the clinic. Using patient BMD to diagnose $\mathrm{OP}$ is based on the hypothesis that BMD is directly related to Young's modulus (the isotropic elastic material property) of bone, and Young's modulus is therefore used as an index of bone strength. However, as only $50-70 \%$ of fractures will be predicted based upon BMD measurements (2), it is clear that BMD values from DXA do not serve as the sole indicators of bone strength, and other important descriptors of bone quality and health (such as micro-architecture and material strength) need to be established,

Corresponding Author: Michael R. Doschak, MSc, PhD; Associate Professor; 2-020J Katz Group Centre for Pharmacy \& Health Research University of Alberta 11361 - 87 Ave. Edmonton, AB, Canada; Email: mdoschak@ualberta.ca 
accepted and factored into the patient assessment scheme.

Both bisphosphonate (BP) and Strontium ranelate (SrR) drug regimens have been shown to reduce the incidence of bone fracture in OP patients in large multicenter clinical trials $(3,4)$, and thus, both drugs are currently indicated for the treatment of OP worldwide $(5,6,7)$. BPs are known to conserve trabecular bone volume and micro-architecture, and therefore bone strength. In contrast, SrR has attracted much attention due to claims of potential dual functionality, with reports of both antiresorptive \& formative events in bone after treatment. Although BP drugs effectively block the resorption of bone, they are well known to interfere with the subsequent phase of coupled osteoblast activity, and reduce bone formation by up to $50 \%(8,9)$. Conversely, studies suggest that $\mathrm{SrR}$ enhances osteoblastic cell function in remodeling and new bone growth $(10,11)$. Subsequent clinical studies indicated that SrR taken over a 3-year period resulted in the heterogeneous distribution of $\mathrm{Sr}$ in bone, resulting in the preservation of bone micro-structure at the tissue level - but not translating into increased stiffness, as measured by indentation modulus (12). Research has evaluated the effectiveness of SrR and BP treatments respectively over an extended period to determine the effect at both clinical and tissue mechanical level (12, 13). However, there remains a lack of data on the chemical composition and material properties of bone tissue when those drugs are administered independently - or potentially in combination.

Hence, the purpose of our study was to; (1) map the distribution and concentration of elemental Calcium, Strontium, and Phosphorus in bone (after BP and/or SrR drug treatments); (2) measure associated BMD and bone micro-architecture (by Micro-CT); (3) assess the material behavior of drugdosed bone (using nano-indentation); and (4) to combine those measurements of bone material property and micro-architecture to assess the outcomes of drug treatments from cohorts of bones in a rat model of developing OP.

\section{METHODS}

\section{Animals and Drug Treatments}

Nineteen 6-month old female Sprague-Dawley rats were obtained from Charles River and maintained at the University of Alberta Biosciences Animal Services Facility. The protocol pertaining to all procedures and aspects of the study was approved by the University of Alberta animal care and ethics committee. Prior to being received at the facility, 16 of the rats were ovariectomized (OVX), while the remaining 3 rats received a sham surgery and the ovaries remained intact. Upon arrival, the OVX rats were divided into 4 groups ( $\mathrm{n}=4$ /group); OVXVehicle (i.e., no drug treatment), OVX-RIS (Risedronate bisphosphonate [BP] treated), OVX-SrR (Strontium Ranelate [Protos $\left.{ }^{\circledR}\right]$ treated), OVXRIS+SrR (combined treatment), and compared against the remaining group of age-matched Shamoperated controls $(n=3)$.

The dosing of Risedronate Bisphosphonate was at $0.06 \mathrm{mg} / \mathrm{kg}$, twice weekly, by subcutaneous injection. That corresponded to an established effective dose for preventing bone loss in this animal model as well as maintaining relevance to the dose currently used in BP treatment of osteoporosis in humans (14). The SrR was dosed at $308 \mathrm{mg} / \mathrm{kg}$ once per day by oral gavage, in order to assure the minimum threshold concentration was achieved to show the potential antiresorptive properties of SrR $(10,15)$, while still trying to assess the SrR function at a concentration that could be regarded as clinically relevant for use in human patients (16).

In rats dosed with RIS + SrR combination therapy, RIS was dosed s.c. 2 hours before giving the oral SrR dose, to allow for RIS to bind bone before strontium entered the system. One $2 \mathrm{~g}$ sachet of commercially available SrR (Servier Laboratories, France) was reconstituted with distilled water and mixed to ensure dissolution. The rats were weighed and the calculated amount of stock solution was dosed daily by oral gavage using a gastric feeding needle. Those rats were fasted for two hours before and two hours after the dose to prevent any food interaction with calcium and any other elements within the feed. After 16 weeks of treatment the rats were euthanized, and the L4, L5 and L6 were vertebral bones dissected fresh and cleaned of soft tissues, wrapped with Phosphate Buffer Saline (PBS)-dampened paper towel and stored frozen at $-20^{\circ} \mathrm{C}$ for subsequent analyses.

\section{Micro-CT Imaging}

Dissected L4, L5 and L6 vertebrae were scanned ex vivo by micro-CT (Skyscan 1076, Kontich BE) at $18 \mu \mathrm{m}$ resolution with $70 \mathrm{kV}, 100 \mu \mathrm{A}$ using a $1.0 \mathrm{~mm}$ Aluminum filter. Projected images of the samples were reconstructed using vendor supplied software (Nrecon 1.6.1.5, SkyScan NV, Kontich BE), with 
images oriented in an axial plane of section. A rectangular region of interest (ROI) which was relative to the size of the vertebral body was used to sample a standardized amount of trabecular bone in the vertebral body in order to conduct standard histomorphometric analyses. The dimension of ROIs varied with the size of the vertebral body. The vertebral body growth plates were utilized as anatomical landmarks in order to consistently segment and sample the same region of trabecular bone from all samples. Vendor morphometric software (CTAn 1.10.0.1, SkyScan, Kontich BE) was then used to analyze trabecular bone volume (BV, $\mathrm{mm}^{3}$ ), fractal dimension (FD) (17), trabecular thickness (Tb.Th., $\mathrm{mm}$ ) and trabecular separation (Tb.Sp., $\mathrm{mm}$ ) and tissue mineral density (TMD, $\mathrm{g} / \mathrm{cm}^{3}$ ) (18) against the vendor-supplied bone phantoms of known mineral density $\left(0.25 \mathrm{~g} / \mathrm{cm}^{3}\right.$ and $0.75 \mathrm{~g} / \mathrm{cm}^{3}$ ) in order to calibrate BMD values from Hounsfield units.

\section{Electron Probe Micro-Analysis}

All L4 vertebrae were bisected coronally (Fig. 1) using an Isomet diamond wafer saw (Buehler Ltd., Lake Bluff IL) with the dorsal bone half reserved for subsequent nano-indentation testing. The ventral bone half was defatted in acetone for 2 weeks then dried in a $40^{\circ} \mathrm{C}$ oven and embedded in epoxy (EpoKwick $^{\circledR}$, Buehler Ltd., Lake Bluff IL). Cylindrical molds 1 inch in diameter and height were used with the cut bone surface embedded facing down for fine grinding and polishing $(\sim 0.5 \mu \mathrm{m})$ on an automated lapping plate. Sample surfaces were then coated with 20-30nm thickness Carbon and electron probe microanalysis (EPMA) was undertaken with a Cameca SX100 electron probe (Cameca, Paris FR), equipped with both Wavelength Dispersive Spectroscopy (WDS) and Energy Dispersive Spectroscopy (EDS), using vendor supplied PeakSight 4.1 (Cameca, Paris FR) analysis software. Elemental calcium $(\mathrm{Ca}) \mathrm{K} \alpha$, strontium ( $\mathrm{Sr}$ ) $\mathrm{L} \alpha$ and phosphorus (P) $\mathrm{K} \alpha$ were analyzed in the same bony regions under $2 \mu \mathrm{m}$ resolution, $15 \mathrm{kV}$ and $15 \mathrm{nA}$. The generated map size for one unit was 512 x 512 pixel as $1 \mathrm{~mm} \times 1 \mathrm{~mm}$. Strontianite EPS3 $\left(\mathrm{SrCO}_{3}\right)$ and Apatite_639 $\left(\mathrm{Ca}_{5}\left(\mathrm{PO}_{4}\right)_{3} \mathrm{~F}\right)$ standards were used to standardize subsequent quantitative analyses. PeakSight 4.1 software was employed to calculate elemental $\mathrm{Ca}, \mathrm{Sr}$ and $\mathrm{P}$ weight percent ( $\mathrm{wt} \%$ ) against a reference spectrum, in order to measure both distribution and concentration. Quantitative elemental analysis was conducted after each scan with PAP oxide correction (19) to get quantities of $w t \%$ values from individual samples. Five point measures were randomly sampled and averaged for areas with $\mathrm{Sr}$ (periosteal surfaces of cortical bone) and without $\mathrm{Sr}$ (deeper cortical bone) for quantitative measurements.

\section{Nano-Indentation Testing}

The dorsal vertebral bone half was secured with modeling clay to orient and support the cut surface parallel to the indenter crosshead. Samples were glued to $15 \mathrm{~mm}$ diameter specimen metal disks and surfaces polished to a depth of $15 \mu \mathrm{m}(0.05 \mu \mathrm{m}$ progressively) then cleaned with flowing distilled water under ultrasonic agitation. Nano-indentation testing was conducted using the TI 900 TriboIndenter (Hysitron Inc., Minneapolis MN). We employed a Berkovich Tip for bone indentation testing, requiring conditioning ("tip bashing") on a Quartz sample for 1000 indentations, with subsequent machine compliance completed on aluminum. Shape Area function calibration was also performed on the Quartz sample in order to define the area function for subsequent modulus calculations. The Berkovich Tip (100-200nm radius, with angle from perpendicular to face $=65.35^{\circ}$; diamond material with Young's modulus $=1141 \mathrm{Gpa}$, Poisson ratio $=0.07$ ) was used to make 10 indentations on regions with and without Sr (Figs. $1 b, c$ ). Force control was $8000 \mu \mathrm{N}$ with 5 seconds of loading, 2 seconds holding and 5 seconds unloading. The Reduced modulus $\left(\mathrm{E}_{\mathrm{r}}\right)$ and Hardness $(\mathrm{H})$ of each indentation were calculated from the unloading displacement-force curve based on the Oliver and Pharr method $(20,21) . \mathrm{E}_{\mathrm{r}}$ and $\mathrm{H}$ were calculated from the displacement-force curve in Fig. 2, using the equations below, Eq. (1) - (4), where $\mathrm{P}_{\max }=$ maximum force, $\mathrm{h}_{\max }=\max$ contact depth, $\mathrm{S}=$ the stiffness while reaching the maximum force, $h_{c}=$ the intercept of the slope of the unloading curve with displacement axis, $A\left(h_{c}\right)=$ calibration of shape area function as a function of $h_{c}$.

$\mathrm{S}=(\mathrm{dP} / \mathrm{dh})_{\mathrm{P} \max }$

$\mathrm{h}_{\mathrm{c}}=\mathrm{h}_{\max }-\left(\mathcal{E} \mathrm{P}_{\max } / \mathrm{S}\right)$; for Berkovich geometry $(\mathcal{E}=$ $0.75)$

$\mathrm{E}_{\mathrm{r}}=\mathrm{S} \pi^{1 / 2} /\left(2\left(\mathrm{~A}\left(\mathrm{~h}_{\mathrm{c}}\right)\right)^{1 / 2}\right)$

$\mathrm{H}=\mathrm{P}_{\max } /\left(\mathrm{A}\left(\mathrm{h}_{\mathrm{c}}\right)\right)$

Indented bone regions were referred to the corresponding EPMA-derived elemental distribution maps, in order to compare the depth-force curves on regions of bone with (or without) Sr incorporation. 


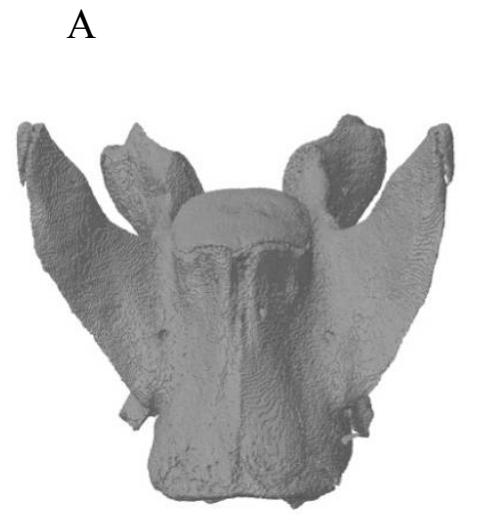

Anterior Posterior(A-P) view
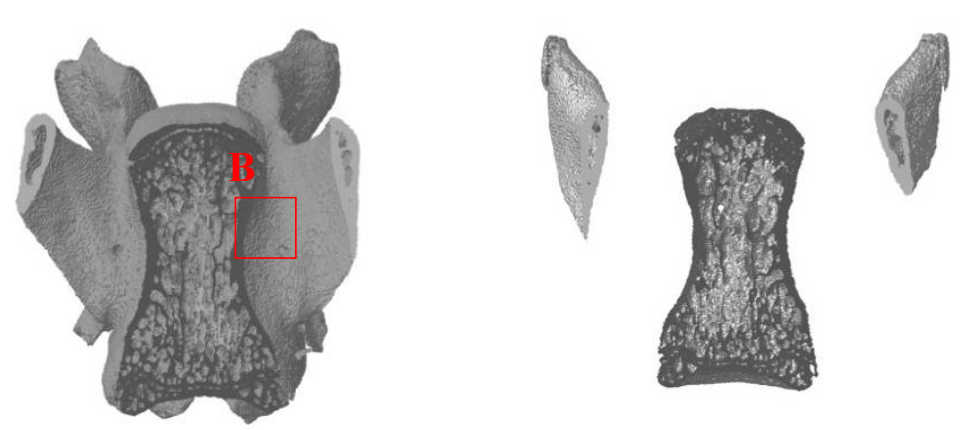

B

Dorsal block part (A-P view) Ventral part (P-A view)

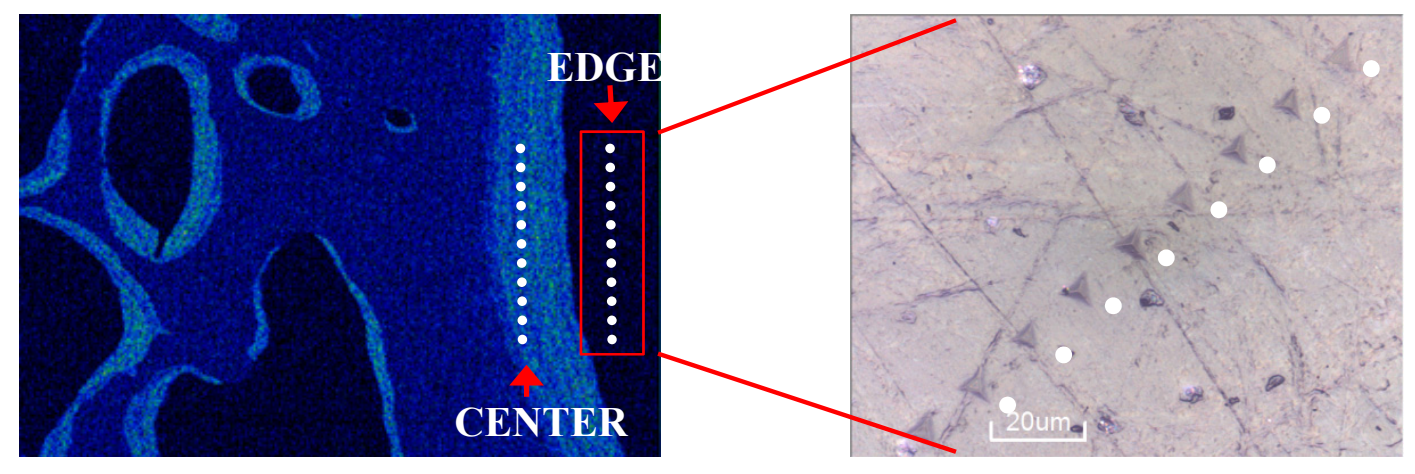

Figure 1. (a) 3-D micro-CT render of rat L4 Vertebral bone, sectioned in the coronal plane. The ventral block was examined by EPMA, with the spatially registered dorsal block used for nano-indentation. (b) Regions of vertebral bone that underwent sequential nano-indentation (line of white dots), either with incorporated Strontium (EDGE), or without (CENTER). The light blue color indicates EPMA detection of elemental strontium. (c). Photomicrograph of triangular nano-indentations (Berkovich tip) on rat vertebral bone surface (Zeiss Axio CSM 700, 100x objective).

\section{Mechanical Testing}

L5 and L6 vertebra were thawed at room temperature, trimmed of bony processes using the diamond wafer saw, with transverse cuts applied to both metaphyseal growth plates. An Instron 4443 materials testing device (Instron, Norwood USA) was used to test L5 and L6 vertebral body in axial compression under displacement control at a speed of $2 \mathrm{~mm} / \mathrm{min}$, to avoid the effect of crosshead impact on the sample prior to failure. Load cell capacity was $\pm 1 \mathrm{kN}$ with displacement recorded according to the position of the crosshead in $\mathrm{mm}$, both with a resolution of 1E-5. Merlin ${ }^{\text {TM }}$ Software and Series IX ${ }^{\mathrm{TM}}$ 8.0.7.0 vendor-supplied software (Instron, Norwood USA) were used to control testing and output the load deformation curve. Since the displacement-force curve were curved before, breaking energy (areas under the displacementforce curve) was more objective to represent physical property of the bone than stiffness. It was then calculated to analyze the difference between treatment groups after BP and/or SrR treatments in the OVX rats. 


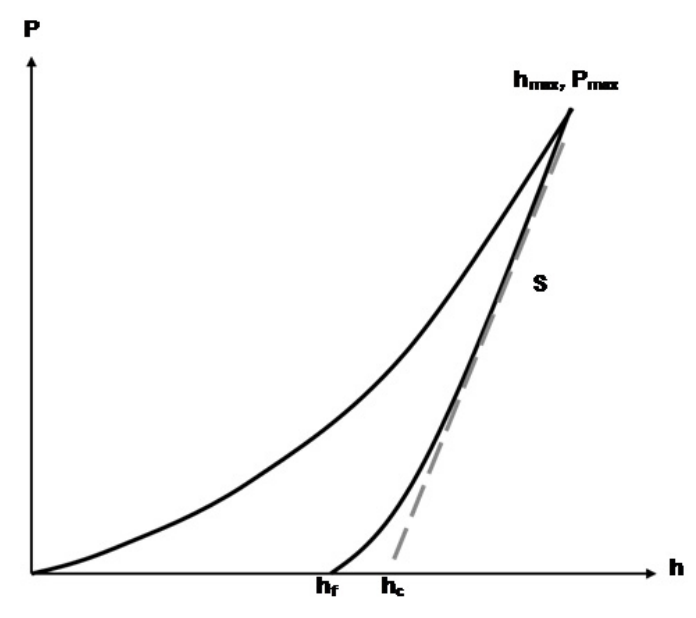

Figure 2. Force( $\mathrm{P})$ - Displacement(h) Curve of nano-indentation tests.

\section{Statistical Analysis}

PASW $^{\circledR}$ Statistics 17.0 was used for statistical evaluations. Due to the small sample numbers, nonparametric Kruskal-Wallis $\mathrm{H}$ test was used to compare differences amongst groups, with the Mann-Whitney $U$ test used to compare between individual groups. An asymptotic value $\leq 0.05$ was used to test for significant differences.

\section{RESULTS}

\section{Structural parameters from Micro-CT}

All drug treatment groups were measured to have significantly greater trabecular bone volume (BV) compared to untreated OVX-Vehicle (Table 1). The 3 -D FD values were increased in treatment groups where BV was also increased. However, we did not measure significant synergistic effects of $\mathrm{BP}+\mathrm{SrR}$ treatments over the BP treatments individually, in either the BV or the FD values. According to the results of FD, SrR treatment increased the complexity of trabecular connectivity in OVX-SrR; however, the complexity was lower than for normal bone. Similarly, we did not measure significant differences in trabecular thickness (Tb.Th.) or BMD between the different treatment groups. From the micro-CT 3-D renders, we examined the trabeculae (FD, Tb.Sp.) and the thickness of cortical bone among groups. It was evident that OVX-RIS and OVX-RIS+SrR dosed animals had significant conservation of trabecular bone volume 4 months after OVX surgery (Fig. 3).

\section{Sr, Ca, P distributions and atomic ratio from EPMA}

From EPMA maps of different groups, we noted that $\mathrm{Ca}$ and $\mathrm{P}$ distributed evenly on the imaged surfaces (Fig. 4). Upon closer examination of individual trabeculae, we noted higher concentrations of $\mathrm{Ca}$ localized centrally in most trabeculae, with lower calcium concentrations localized as a thin layer on the exterior surfaces of both cortical and trabecular bone. Those observations suggested new bone formation events through appositional growth on the periosteal surface of cortical bone, and through remodeling on endosteal trabecular surfaces. Trabecular bone in proximity to the growth plate was also measured to have increased $\mathrm{Ca}$ concentration. Of particular significance was the dynamic localization of elemental Sr (using EPMA) to bone turnover in this animal model of OP (Fig. 5). The periosteal cortical bone in both the OVX-SrR and OVX-RIS+SrR groups exhibited layers of Sr-enriched appositional bone growth in the order of $50-100 \mu \mathrm{m}$ over the 16 weeks period. $\mathrm{Sr}$ also deposited in trabecular bone distal to the growth plate (i.e., at the primary spongiosum). In EPMA maps of OVX-SrR, $\mathrm{Sr}$ incorporation encompassed individual trabeculae throughout the marrow cavity, to a depth of approximately $20 \mu \mathrm{m}$. In stark contrast, $\mathrm{Sr}$ deposition was significantly reduced in the presence of the BP drug (i.e., in the OVX-RIS+SrR group), both in the number of formative events as well as in the depth of mineral deposition compared to $\mathrm{SrR}$ treatment alone. Quantitative analysis measured reduced $\mathrm{wt} \%$ values in the OVX-RIS+SrR treated group for $\mathrm{P} 2 \mathrm{O} 5, \mathrm{CaO}$ and $\mathrm{SrO}$ (48-80\% of normal). In the Sr-free groups (i.e., Sham, OVX-Vehicle, OVX-RIS), no significant quantity of elemental $\mathrm{Sr}$ was measured. The $\mathrm{Ca}$ or $\mathrm{Ca}+\mathrm{Sr}$ and $\mathrm{P}$ concentrations always maintained a consistent ratio. The atomic ratio $(\mathrm{Ca} / \mathrm{P}$ or $\mathrm{Ca}+\mathrm{Sr} / \mathrm{P})$ was 1.66 in all groups (Table 1). The examined average $\mathrm{wt} \%$ of $\mathrm{Ca}$ is $29 \%$ and $14 \%$ for $\mathrm{P}$. There was no significant difference of the $\mathrm{Ca} / \mathrm{P}$ ratio among non-Sr treatment groups. In both $\mathrm{Sr}$ treated groups (OVX-SrR and OVX-RIS+SrR), we measured $2.4 \%-6.6 \%$ lower atomic ratio of $(\mathrm{Ca}+\mathrm{Sr}) / \mathrm{P}$ in comparison with non$\mathrm{Sr}$ groups. Accordingly, our data indicated that the wt $\%$ of Sr after 16 weeks of dosing with SrR (at the 
dosage employed) resulted in less than $2 \%$ elemental $\mathrm{Sr}$ incorporation into the bone of the OVX-SrR and OVX-RIS+SrR groups.

\section{Depth-Force curves from Nano-Indentation tests}

Using nano-indentation, we did not measure any differences in reduced modulus or hardness after 16 weeks of drug treatment at the drug dosages employed in this study. We also compared forcedisplacement curves of nano-indentation on areas shown by EPMA with or without $\mathrm{Sr}$ (EDGE/CENTER of cortical bone) (Fig. 1b) relative to each group. The curves showed there were no significant differences among groups at areas of CENTER cortical bone under the given indentation conditions. We noted a non-significant trend of OVX-RIS samples exhibiting increased contact depth under the standardized loading conditions compared to all other treatment groups (Fig. 6).

\section{Mechanical Testing}

In contrast to nano-indentation, the breaking energy of global vertebral body compression in L5 and L6 showed that both BP treated groups had increased structural bone strength compared with all other groups (Fig. 7). Both OVX-RIS and OVX-RIS+SrR dosed animals showed improved breaking energy compared to the other OVX groups (OVX-Vehicle and OVX -SrR).

Table 1.Data of micro-CT, EPMA, nano-indentation and mechanical test

\begin{tabular}{|c|c|c|c|c|c|}
\hline \multicolumn{6}{|c|}{ Treatment } \\
\hline & Sham & OVX-Vehicle & OVX-RIS & OVX-SrR & OVX-RIS+SrR \\
\hline \multicolumn{6}{|c|}{ micro-CT parameters of the average of $L 4, L 5, L 6$} \\
\hline $\mathrm{BV}\left(\mathrm{mm}^{3}\right)$ & $153+11^{\mathrm{b}, \mathrm{e}}$ & $133 \pm 10^{\mathrm{a}, \mathrm{c}, \mathrm{d}, \mathrm{e}}$ & $169 \pm 22^{b, d}$ & $145 \pm 2^{\mathrm{b}, \mathrm{c}, \mathrm{e}}$ & $172 \pm 8^{\mathrm{a}, \mathrm{b}, \mathrm{d}}$ \\
\hline $\operatorname{TMD}\left(\mathrm{g} / \mathrm{cm}^{3}\right)$ & $0.65 \pm 0.05$ & $0.64 \pm 0.01$ & $0.67 \pm 0.02$ & $0.65 \pm 0.02$ & $0.65 \pm 0.01$ \\
\hline FD & $2.48 \pm 0.04^{\mathrm{b}, \mathrm{d}}$ & $2.36 \pm 0.03^{\mathrm{a}, \mathrm{c}, \mathrm{e}}$ & $2.53 \pm 0.03^{\mathrm{b}, \mathrm{d}}$ & $2.41 \pm 0.02^{\mathrm{a}, \mathrm{c}, \mathrm{e}}$ & $2.54 \pm 0.02^{\mathrm{b}, \mathrm{d}}$ \\
\hline Tb.Th. (mm) & $0.13 \pm 0.02$ & $0.11 \pm 0.01$ & $0.13 \pm 0.01$ & $0.12 \pm 0.01$ & $0.12 \pm 0.01$ \\
\hline Tb.Sp. (mm) & $0.22 \pm 0.02^{\mathrm{b}, \mathrm{d}}$ & $0.32 \pm 0.05^{\mathrm{a}, \mathrm{c}, \mathrm{e}}$ & $0.21 \pm 0.02^{\mathrm{b}, \mathrm{d}}$ & $0.29 \pm 0.03^{\mathrm{a}, \mathrm{c}, \mathrm{e}}$ & $0.22 \pm 0.01^{\mathrm{b}, \mathrm{d}}$ \\
\hline \multicolumn{6}{|c|}{ EPMA atomic ratio of $S r, C a$ and,P at $S r$ deposition region } \\
\hline$(\mathrm{Sr}+\mathrm{Ca}) / \mathrm{P}$ & $1.65 \pm 0.01^{\mathrm{d}, \mathrm{e}}$ & $1.65 \pm 0.01^{\mathrm{d}, \mathrm{e}}$ & $1.66 \pm 0.01^{\mathrm{d}, \mathrm{e}}$ & $1.61 \pm 0.02^{\mathrm{a}, \mathrm{b}, \mathrm{c}}$ & $1.54 \pm 0.10^{\mathrm{a}, \mathrm{b}, \mathrm{c}}$ \\
\hline $\mathrm{Sr} / \mathrm{P}-\mathrm{HSr}$ & $0.00<0.00^{\mathrm{d}, \mathrm{e}}$ & $0.00<0.00^{\mathrm{d}, \mathrm{e}}$ & $0.00<0.00^{\mathrm{d}, \mathrm{e}}$ & $0.03<0.00^{\mathrm{a}, \mathrm{b}, \mathrm{c}}$ & $0.03< \pm 0.00^{\mathrm{a}, \mathrm{b}, \mathrm{c}}$ \\
\hline \multicolumn{6}{|c|}{ Nanoindentation results of Reduced Modulus(Er) and Hardness(H) } \\
\hline $\operatorname{Er}(\mathrm{GPa})-\mathrm{EDGE}$ & $31.52 \pm 6.62$ & $31.78 \pm 3.68$ & $23.82 \pm 3.80$ & $31.10 \pm 6.85$ & $29.85 \pm 5.73$ \\
\hline Er(GPa)-CENTER & $33.02 \pm 2.90$ & $32.63 \pm 3.53$ & $30.55 \pm 5.45$ & $36.28 \pm 2.10$ & $33.78 \pm 1.26$ \\
\hline $\mathrm{H}(\mathrm{GPa})-\mathrm{EDGE}$ & $1.57 \pm 0.56$ & $1.62 \pm 0.39$ & $1.20 \pm 0.38$ & $1.48 \pm 0.14$ & $1.52 \pm 0.31$ \\
\hline H(GPa)-CENTER & $1.62 \pm 0.17$ & $1.61 \pm 0.14$ & $1.42 \pm 0.47$ & $1.96 \pm 0.34$ & $1.73 \pm 0.14$ \\
\hline \multicolumn{6}{|c|}{ Mechanical Testing for Breaking Energy of L5,L6 } \\
\hline Breaking Energy (mm-kN) & $0.19 \pm 0.01$ & $0.18 \pm 0.04$ & $0.23 \pm 0.07$ & $0.19 \pm 0.01$ & $0.27 \pm 0.03$ \\
\hline
\end{tabular}

Data expressed as Mean \pm SD. ${ }^{\mathrm{a}}$ Significantly different from Sham. ${ }^{\mathrm{b}}$ Significantly different from OVX-Vehicle. ${ }^{\mathrm{c}}$ Significantly different from OVX-RIS. ${ }^{\mathrm{d}}$ Significantly different from OVX-SrR. ${ }^{\mathrm{e}}$ Significantly different from OVXRIS+SrR.

Abbreviations: BV: Bone Volume; TMD: Tissue Mineral Density; FD: Fractal Dimension; Tb.Th: Trabecular Thickness; Tb.Sp.: Trabecular Separation. 


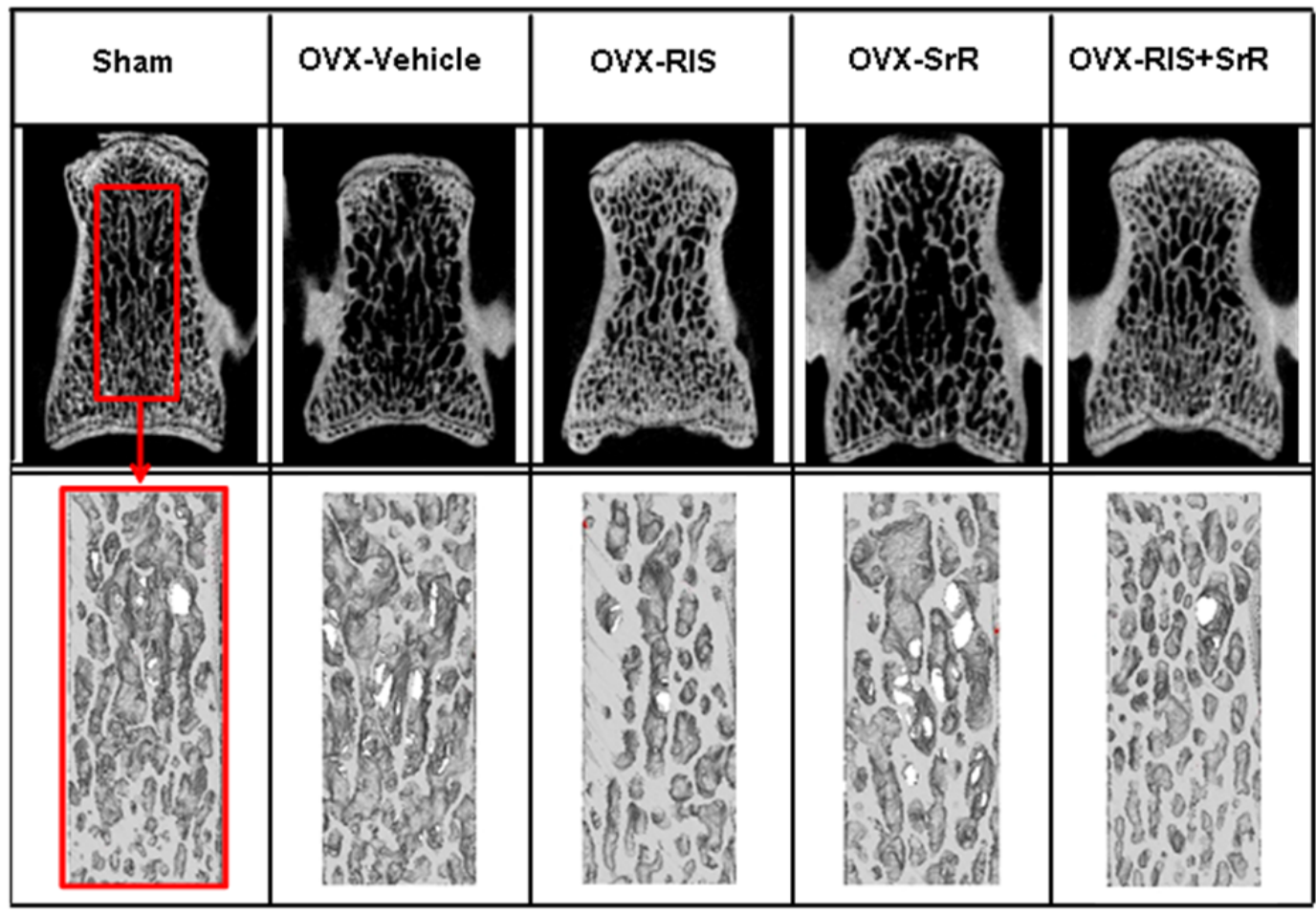

Figure 3. Upper panel: Cross-sectional images of Micro-CT in the coronal plane of L4 vertebral bodies. Lower panel: 3Dimensional renders of trabecular bone from a rectangular region of interest.

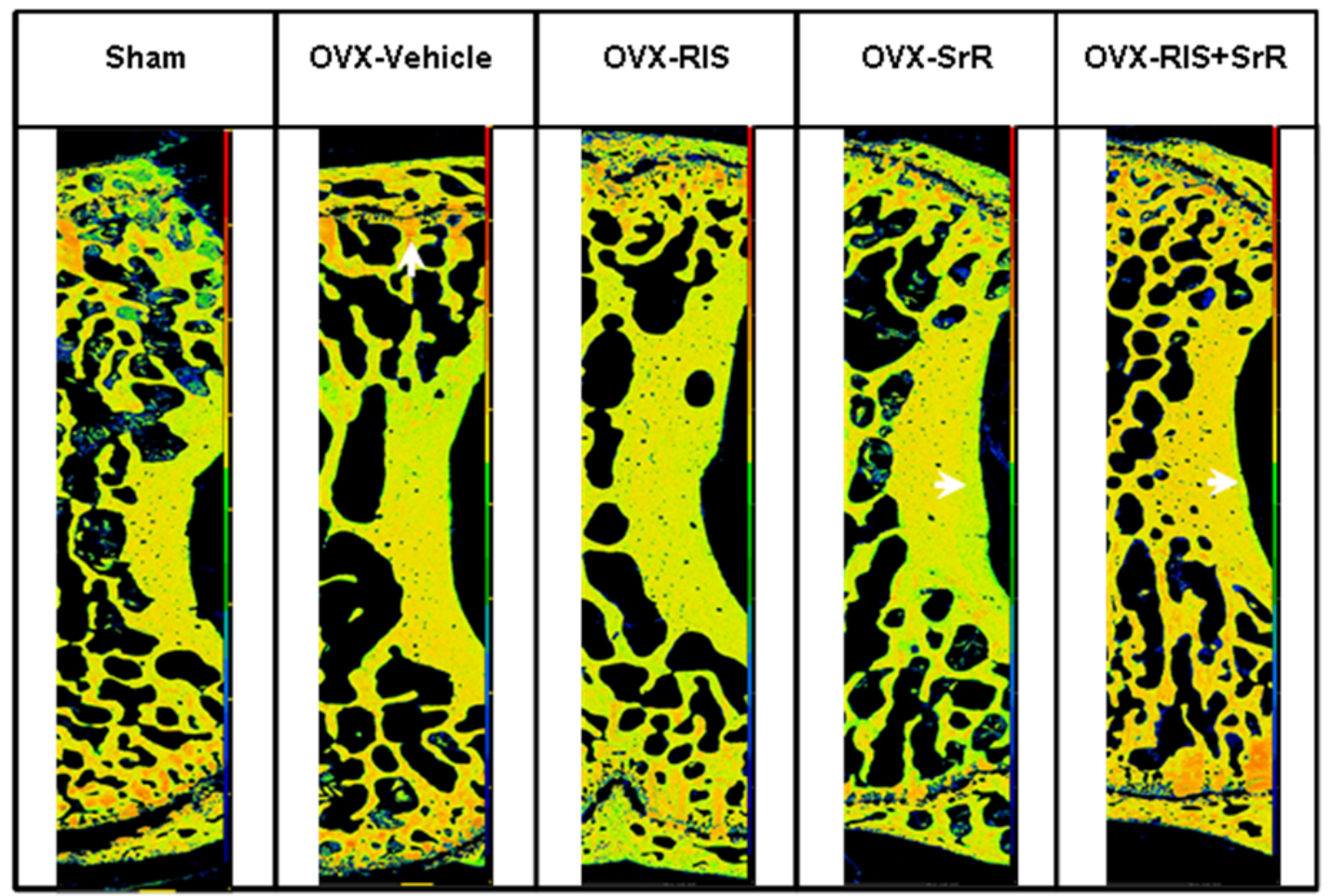

Figure 4. Electron Probe Micro-Analysis (EPMA) maps of Calcium Distribution (Anterior-Posterior view of the half Vertebrae Body). Warmer colors indicate increased concentrations of elemental calcium. 


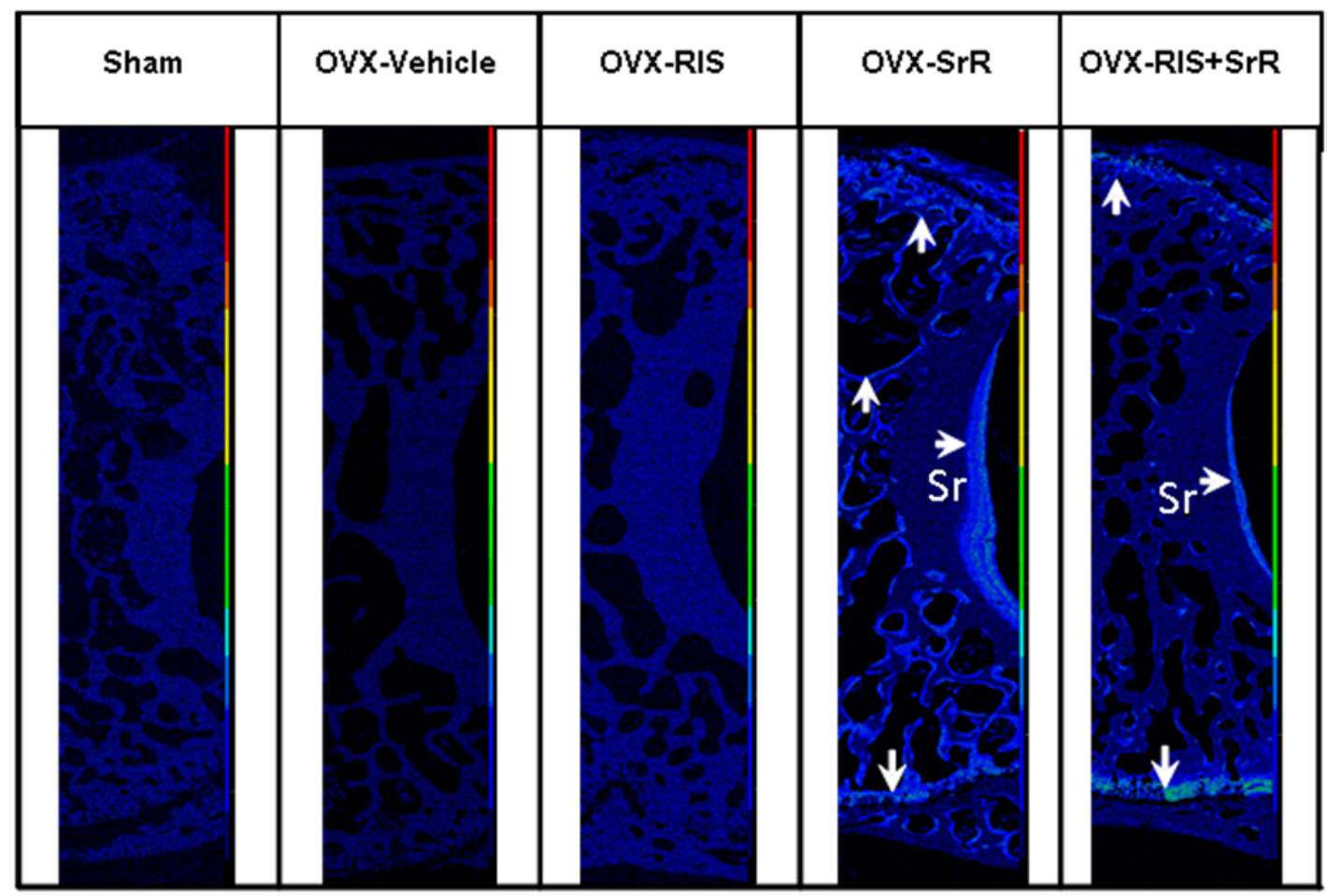

Figure 5. Electron Probe Micro-Analysis (EPMA) maps of elemental strontium distribution (Coronal section of rat L4 vertebral body). The light blue color indicates higher concentrations of elemental strontium.

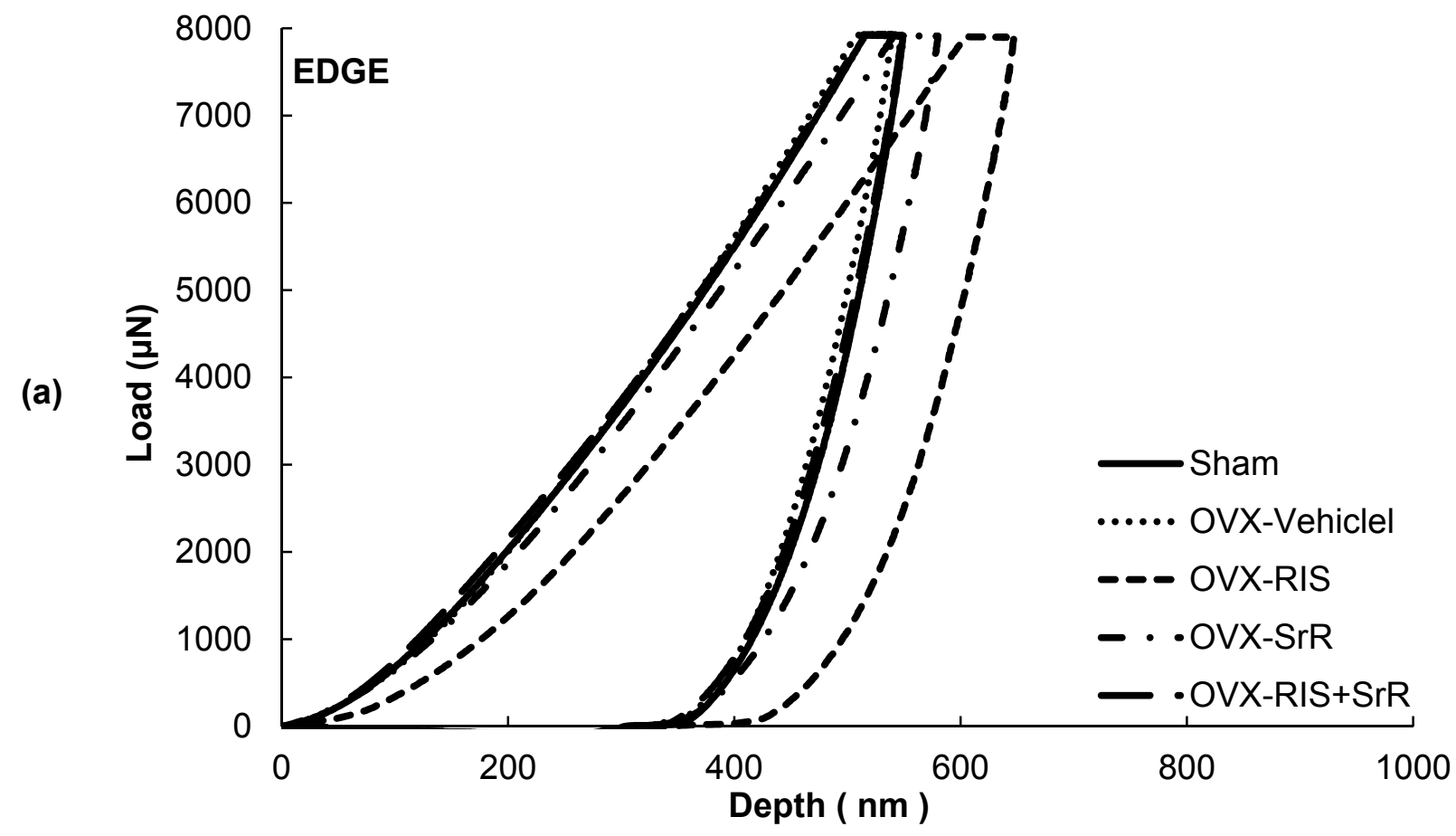




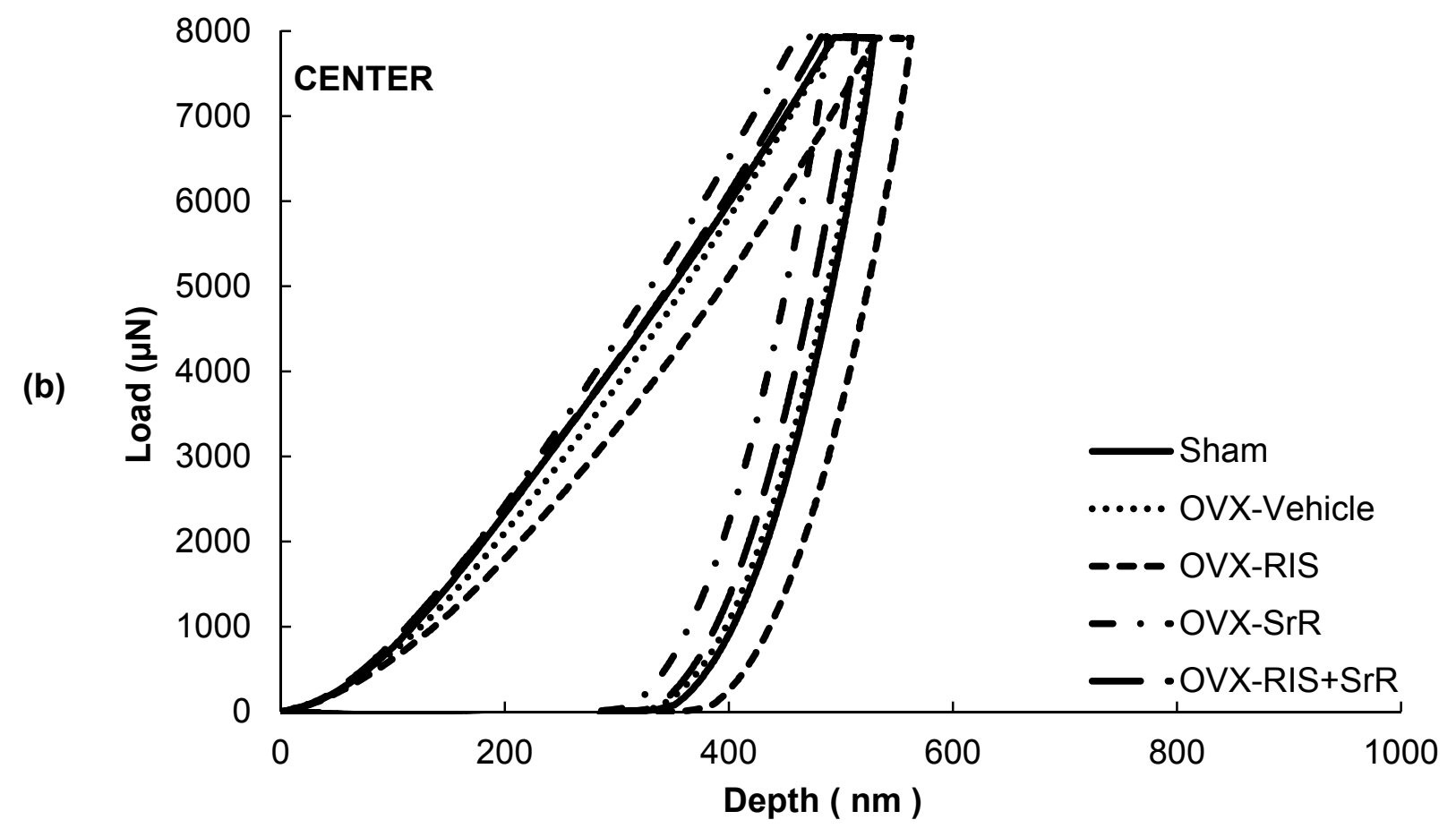

Figure 6. Depth $(\mathrm{nm})$ vs. Load $(\mu \mathrm{N})$ curves from nano-indentation tests of different groups. Curves of different groups are represented by different colors. a) Curves from indenting on EDGE. OVX-RIS shows a trend towards higher contact depth under the same indenting condition. b) Curves from indenting on CENTER. There were no apparent differences in contact depth between the treatments in the CENTER region of older, non-remodeling bone.

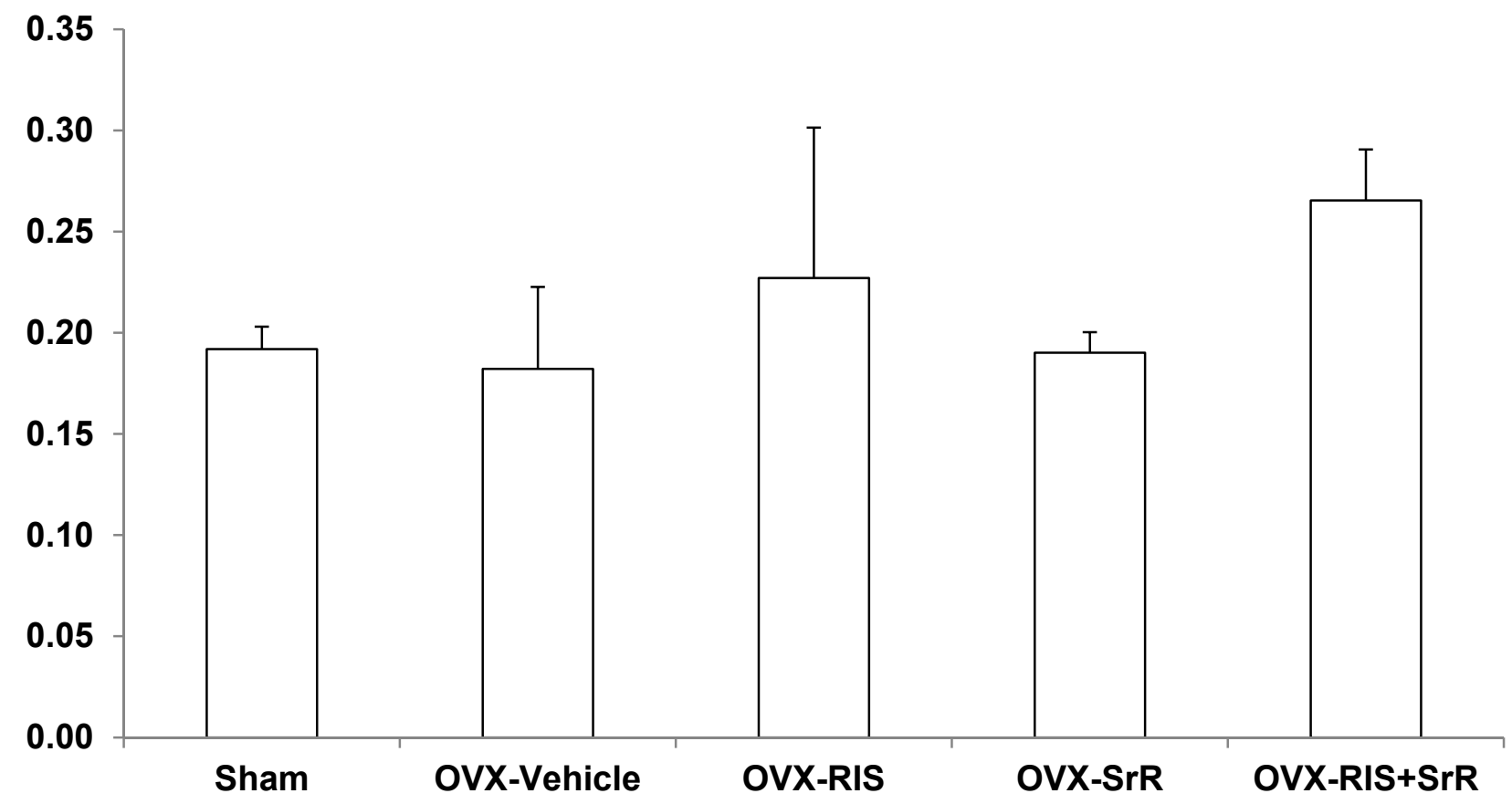

Figure 7. The Breaking Energy of Rat Vertebrae L5, L6 tested under load (in compression) to failure. 


\section{DISCUSSION}

Bone fracture is a serious health concern with postmenopausal osteoporosis. Current BP antiresorptive drug interventions are highly effective at the conservation of existing bone volume, trabecular micro-architecture (and structural strength respectively), thereby preventing OP-related fracture during therapy. In our study, it was clear that the BP-treatment of OVX rats resulted in the significant conservation of bone volume and micro-architectural quality over all other treatment groups, which further translated into increased structural breaking energy during mechanical loading in compression.

However, an extensive period of BP drug treatment may also introduce alterations in the remodeling (i.e., replenishing) ability of bone in the long term (22). Recent studies have reported the unexpected occurrence of spontaneous atypical subtrochanteric and diaphyseal femoral fractures in OP patients after long-term BP treatment (23), and those reports were reviewed by an ASBMR appointed task force (24). They concluded that the incidence of fracture associated with BP therapy for osteoporosis appears to be very low, and that a causal association has not been established. Amongst other recommendations, however, they encouraged further research in animal models to gather additional data to establish the true risk of BP use associated with conditions of catastrophic structural bone failure, which serves as a rationale for our current investigation.

In contrast to BP drug therapy, SrR treatment (at the dose tested) did not increase the structural breaking energy of vertebral bone during mechanical loading in compression - despite showing some ability in the preservation of trabecular bone volume by ex vivo micro-CT. Those results agreed with published clinical findings showing that SrR taken over a 3-year period resulted in the preservation of bone microstructure at the tissue level, but did not translate into increased bone strength, as measured by indentation modulus (12). They also agreed with results by Bain et al who measured that bone stiffness in SrRtreated animals was unchanged from values measured in OVX animals treated with vehicle despite measuring the contrasting outcomes of dose-dependent higher maximal load, and the significant conservation of maximal energy to failure compared to OVX-untreated animals (25).

Hence, the biological mechanism whereby SrR contributes to improve bone strength and antifracture efficacy in clinical trials is still not entirely understood. Recent research by Boyd et al measured improved bone micro-architecture and reported an increase in stiffness in SrR treated rats as compared to controls (26). In that research, dosing with SrR commenced in growing, juvenile (i.e., $16 \mathrm{wk}$ ) normal Wistar rats, with a SrR dosage of $900 \mathrm{mg} / \mathrm{kg} / \mathrm{day}$ for $104 \mathrm{wks}$. They measured that SrR-treated rats did not show a decrease in bone strength compared to age-matched normal controls. However, it is difficult to compare those results to our study as we employed 6-month mature OVX Sprague-Dawley rats, with a significantly reduced dosage and duration of $\mathrm{SrR}(308 \mathrm{mg} / \mathrm{kg} /$ day for 16 weeks) which nonetheless was previously reported to be at an effective dose range to effect both antiresorptive and anabolic activity $(11,15)$.

Our EPMA findings confirmed that in the normal rat, the mineralized bone matrix is heterogeneous in material composition with only trace amounts of elemental $\mathrm{Sr}$ uptake from the diet. In stark contrast, after SrR treatment, the $\mathrm{Sr}$ atom serves as a surrogate for elemental $\mathrm{Ca}$ uptake and was rapidly and heavily incorporated at sites of newly mineralizing bone. Those observations agreed with previously published reports on the incorporation and distribution of $\mathrm{Sr}$ in bone (27). In our study, profound regional differences in elemental composition were sensitively mapped to periosteal cortical and endosteal trabecular bone surfaces. Despite the significant increase in elemental $\mathrm{Sr}$ deposited in those regions of mineralized bone matrix, our mechanical testing evaluations of those same defined regions of Srenriched bone using nano-indentation did not measure significant differences in bone tissue modulus, albeit confined to the SrR dosage we tested.

The 4 month duration of SrR drug administration in our study was significantly less than that used in the clinical treatment of human patients with osteoporosis. Nonetheless, the $2 \mathrm{wt} \%$ of $\mathrm{Sr}$ deposition we measured was in accord with $\mathrm{Sr}$ deposition attained by taking higher dosages and treating patients for 3 years (12). Previous studies have concluded that $\mathrm{SrR}$ was effective in preventing rat OVX-induced bone loss and improving bone mechanical strength in doses as low as 125 
$\mathrm{mg} / \mathrm{kg} /$ day (11). In contrast, Fuchs et al suggested that a SrR dosage of $25 \mathrm{mg} / \mathrm{kg} /$ day in the rat was comparable to the human dosage of $2 \mathrm{~g}$ /day on a $\mathrm{mg} / \mathrm{kg}$ basis. Their study also confirmed the $25 \mathrm{mg} / \mathrm{kg} /$ day dose levels produced serum levels of strontium similar to those found clinically (15), but they were not able to confirm the anabolic effect of SrR treatment in the OVX rat at the $25 \mathrm{mg} / \mathrm{kg} /$ day dose. In a letter to the editor regarding that study, the authors were challenged in that the dose of SrR used was likely too low (i.e., sub-therapeutic)(28), and referred to the dose of $308 \mathrm{mg} / \mathrm{kg} /$ day in an earlier published study as an effective dose that increased the trabecular bone volume by $30-36 \%$ (15). Indeed, in a more recent paper, $900 \mathrm{mg} / \mathrm{kg} /$ day was used as a SrR dosage in rats to increase bone resistance and promote bone forming activity (26). That dose was stated to correspond to twice the human serum circulating level in rats based on pharmacokinetic measurements, but those statements were not supported by the evaluations reported in that paper. If an average postmenopausal osteoporosis patient is $70 \mathrm{~kg}$, on a $\mathrm{mg} / \mathrm{kg}$ basis that would translate to $63 \mathrm{~g}$ of SrR per day. Thus, clearly, establishing clarity regarding the accuracy of pharmacokinetic and pharmacodynamic parameters surrounding SrR usage is of critical importance, as the current human clinical dose remains at the $2 \mathrm{~g}$ SrR p.o. once daily regimen (15).

With our in vivo micro-CT analyses, we accurately measured the loss of trabecular bone mass and micro-architectural connectivity in vertebrae from untreated OVX animals developing OP. We found that $\mathrm{SrR}$ at the dose of 308 $\mathrm{mg} / \mathrm{kg} /$ day over 4 months significantly conserved vertebral trabecular bone volume compared to untreated OVX controls, however remained significantly less potent than OVX-RIS treated animals. Namely, in all BP treatment groups, we measured the significant conservation of BV, FD and Tb.Sp. compared to both untreated OVX controls and OVX-SrR dosed animals. Our bone mineral density (BMD) measurements did not show significant differences between any of the treatments, including that of SrR monotherapy (Table 1). In addition, we measured significantly reduced global (i.e., the entire bone sample) mechanical strength of vertebral bone in compression to failure in OVX rats, and the BP drug RIS (either as a monotherapy, or in combination with $\mathrm{SrR}$ ) significantly improved the mechanical strength of OVX vertebral bone, whereas $\mathrm{SrR}$ was unable to significantly impact bone mechanical strength.

Despite the ability for RIS treatment to preserve existing bone mass and micro-architectural structure, remodeled bone formation frequency and duration was significantly reduced in OVX-RIS dosed rats co-administered SrR, compared to OVXSrR dosed rats, as evidenced by EPMA analysis of incorporated elemental Sr. Thus, for remodeling BP-dosed bone, the significant reduction in trabecular matrix formation may alter the local volume and material composition of bone. In turn, that would potentially influence the anti-fracture strength, particularly if associated with thinning trabecular bone.

One surprising finding of our study was the non-significant trend of OVX-RIS samples exhibiting increased contact depth under nanoindentation compared to all other treatment groups. Clearly, BP drugs are known to increase global tissue modulus in great part through the conservation of bone volume and microarchitectural connectivity, which serve to stiffen the bone in response to loading. However, it is important to note that our nano-indentation measurements were conducted in regions of newly formed bone (i.e., mineralized osteoid) that incorporated significant amounts of $\mathrm{BP}, \mathrm{SrR}$, or a combination of those drug molecules. Thus, the localized presence of those drugs likely influenced cellular biology and bone matrix formation in a "site-specific" manner.

There are several limitations of this preliminary study. Firstly, the sample number per treatment group remained small, due to the complexity and expense of coordinating precise regional analyses of drug-dosed bone regions between the micro-CT, EPMA and nano-indentation techniques that were employed. However, as our study purpose was to contrast the material and compositional make-up of bone after BP and/or SrR drug therapy in an animal model of OP, our findings should remain of interest. Another study limitation was that our nanoindentation facility is limited to the indention of dry bone samples. Therefore, the depth-displacement curves of dried samples will likely be stiffer than that measured with hydrated samples, although bone samples from each group were treated and dried using the same procedure. Thus, comparison of our measured values to those from other facilities 
may differ in magnitude, as the procedure for sample preparation, testing and handling will differ.

We conclude that BP drugs dominate the conservation of trabecular geometry and corresponding structural bone strength in OP rats, whereas SrR may influence bone volume and material composition locally. The administration of $\mathrm{SrR}$ and the subsequent uptake of $\mathrm{Sr}$ appeared to have less of an impact on the structural integrity of bone as it did on the utility of its presence in permitting new bone formation during the remodeling cycle. The incorporation of BP and/or Sr drugs (at the dosages used) did not increase the mechanical stiffness of bone matrix in locations known to contain those drugs, and remained similar to values measured in sham-operated controls.

Our study further highlights the potential that $\mathrm{Sr}$ drugs offer for use as exquisite indicators of bone turnover. Perhaps it is the non-ionizing, tracer potential observed when administering subtherapeutic quantities of SrR that could prove of greatest utility for clinicians in the assessment and understanding of regional bone turnover for individual patients. Further studies will help shed light on the diagnostic tracer utility of $\mathrm{Sr}$ based compounds that may eventually find use as effective clinical diagnostic tools in the diagnosis and treatment of bone injury, repair and disease.

\section{ACKNOWLEDGMENTS}

The authors thank Michael D. Jones and Colin T. Lee for help with drug dosing, and John Simon for help with preparation of the manuscript. We also thank Sergei Matveev for assistance with the EPMA analyses and Eric Flaim for assistance with the Hysitron Triboindenter.

This research was funded by The (Canadian) Arthritis Society (TAS), and by the OA Alberta Team Grant from the Alberta Heritage Foundation for Medical Research (AHFMR).

Authors' roles: Study design: YW, SMA, MJD and MRD. Study conduct: YW, and MRD. Data collection: YW, and DM-P. Data analysis: YW, DM-P, and MRD. Data interpretation: YW, and MRD. Drafting manuscript: YW, and MRD. Revising manuscript content: YW, SMA, MJD, DM-P, and MRD. Approving final version of manuscript: YW, SMA, MJD, DM-P, and MRD. MRD takes responsibility for the integrity of the data analysis.

\section{REFERENCES}

1. de Villiers TJ. Bone health and osteoporosis in postmenopausal women. Best Pract Res Clin Obstet Gynaecol, 2009; 23:73-85.

2. Griffiths J. Raman spectroscopy for medical diagnosis. Anal Chem, 2007; 79:3975-3978.

3. Reginster J, Minne HW, Sorensen $\mathrm{OH}$ et al. Randomized trial of the effects of risedronate on vertebral fractures in women with established postmenopausal osteoporosis. Vertebral Efficacy with Risedronate Therapy (VERT) Study Group. Osteoporos Int, 2000; 11:83-91.

4. Meunier PJ, Roux C, et al. The effects of strontium ranelate on the risk of vertebral fracture in women with postmenopausal osteoporosis. N Engl J Med, 2004; 350:459-468.

5. Borgström F, Ström O, Coelho J et al. The costeffectiveness of strontium ranelate in the uk for the management of osteoporosis. Osteoporos Int, 2010; 21:339-349.

6. Hiligsmann $M$, Bruyère $\mathrm{O}$, Reginster JY. Costeffectiveness of strontium ranelate versus risedronate in the treatment of postmenopausal osteoporotic women aged over 75 years. Bone, 2010; 46:440-446.

7. Hwang JS, Chen JF, Yang TS et al. The effects of strontium ranelate in asian women with postmenopausal osteoporosis. Calcif Tissue Int, 2008; 83:308-314.

8. Reszka AA, Rodan GA. Nitrogen-containing bisphosphonate mechanism of action. Mini Rev Med Chem, 2004; 4:711-719.

9. Iwata K, Li J, Follet H, Phipps RJ, Burr DB. Bisphosphonates suppress periosteal osteoblast activity independently of resorption in rat femur and tibia. Bone, 2006; 39:1053-1058.

10. Bonnelye E, Chabadel A, Saltel F, Jurdic P. Dual effect of strontium ranelate: stimulation of osteoblast differentiation and inhibition of osteoclast formation and resorption in vitro. Bone, 2008; 42:129-138.

11. Ammann P, Shen V, Robin B, Mauras Y, Bonjour J P, Rizzoli R. Strontium ranelate improves bone resistance by increasing bone mass and improving architecture in intact female rats. J Bone Miner Res, 2004; 19:2012- 2020.

12. Roschger P, Manjubala I, Zoeger $\mathrm{N}$ et al. Bone material quality in transiliac bone biopsies of postmenopausal osteoporotic women after 3 years of strontium ranelate treatment. J Bone Miner Res, 2010; 25:891-900.

13. Muscoso E, Puglisi N, Mamazza $\mathrm{C}$ et al. Antiresorption therapy and reduction in fracture susceptibility in the osteoporotic elderly patient: open study. Eur Rev Med Pharmacol Sci, 2004; 
8:97- 102 .

14. Campbell GM, Bernhardt R, Scharnweber D, Boyd SK. The bone architecture is enhanced with combined PTH and alendronate treatment compared to monotherapy while maintaining the state of surface mineralization in the OVX rat. Bone, 2011; 49(2):225-232.

15. Marie PJ, Hott M, Modrowski $D$ et al. An uncoupling agent containing strontium prevents bone loss by depressing bone resorption and maintaining bone formation in estrogen-deficient rats. J Bone Miner Res, 1993; 8:607-615.

16. Fuchs RK, Allen MR, Condon KW et al. Strontium ranelate does not stimulate bone formation in ovariectomized rats. Osteoporos Int, 2008; 19:13311341.

17. Chappard D, Legrand E, Haettich B. et al. Fractal dimension of trabecular bone: comparison of three histomorphometric computed techniques for measuring the architectural two-dimensional complexity. J. Pathol. 2001; 195: 515-521.

18. Bouxsein ML, Boyd SK, Christiansen BA etal. Guidelines for Assessment of Bone Microstructure in Rodents Using Micro-Computed Tomography. J Bone Miner Res, 2010; 25: 1468-1486.

19. Pouchou JL, Pichoir F. "PAP“(phi-rho-z) procedure for improved quantitative microanalysis. In: Armstrong JT,editor. Microbeam Analysis. San Francisco Press: San Francisco, 1985; 104-106.

20. Fischer-Cripps AC. Introduction to contact mechanics, 2nd ed. Springer,New York, pp 221, 2007

21. Fischer-Cripps AC. Nanoindentation, $2^{\text {nd }}$ ed.
Springer,New York, pp 197, 2004.

22. Seeman E. To stop or not to stop, that is the question. Osteoporos Int, 2009; 20:187-195.

23. Ali T, Jay RH. Spontaneous femoral shaft fracture after long-term alendronate. Age Ageing, 2009; 38(5):625-626.

24. Shane E, Burr D, Ebeling PR et al. Atypical subtrochanteric and diaphyseal femoral fractures: report of a task force of the American Society for Bone and Mineral Research. J Bone Miner Res, 2010; 25:2267-2294.

25. Bain SD, Jerome C, Shen V, Dupin-Roger I, Ammann P. Strontium ranelate improves bone strength in ovariectomized rat by positively influencing bone resistance determinants. Osteoporos Int, 2009; 20:1417-1428.

26. Boyd SK, Szabo E, Ammann P. Increased bone strength is associated with improved bone microarchitecture in intact female rats treated with strontium Ranelate: A finite element analysis study. Bone, 2011; 48:1109-1116.

27. Boivin G, Farlay D, Khebbab M T, Jaurand X, Delmas P D, Meunier P J. In osteoporotic women treated with strontium ranelate, strontium is located in bone formed during treatment with a maintained degree of mineralization. Osteoporos Int, 2010; 21:667- 677.

28. Marie PJ. Effective doses for strontium ranelate. Osteoporos Int, 2008; 19:1813.

29. Systematic Treatment after Successful Surgical Treatment for Primary Hyperparathyroidism with Strontium Ranelate. ClinicalTrials.gov Identifier: NCT01222026. Medical University of Vienna. 